\title{
4D Printing and Characterization of Shape Memory Polymer (SMP) Based Smart Gripper
}

\author{
Francis Irungu Maina, Nahashon Osinde, Japheth Ka'pesha Odira, Patrick Kariuki Wanjiru, \\ and Margaret Wanjiku Mwangi
}

\begin{abstract}
Shape Memory Polymer (SMP) is stimuliresponsive material with the ability to recover the original shape from a deformation upon triggering by an appropriate stimulus like heat, light, and electricity. The shape recovery properties can be harnessed through $4 \mathrm{D}$ printing of selfrecoverable functional structures and made usable in fields like medicine and robotics. To investigate the recovery properties, best printing parameters and optimal sizes, $4 \mathrm{D}$ reconfigurable gripper designed in CAD was printed in Ultimaker 2 Printer. Different stencils were made in varying printing parameters of temperature, infill, speed and time. Analysis for the stencils proved best print quality at a temperature of $195^{\circ} \mathrm{C}$ and nozzle retract speed of $40 \mathrm{~mm} / \mathrm{s}$. Shape recovery characterization was done on MATLAB. A printing temperature of $203{ }^{\circ} \mathrm{C}$, infill density of $38 \%$ and printing speed of $40 \mathrm{~mm} / \mathrm{s}$ gave the gripper with the best print quality. Characterization of the varying performances of the four grippers was attributed to the different infill percentages. The lower the infill, the higher the recovery rate due to the low stiffness of the gripper. The best recovery rate of $96.93 \%$ was associated with an optimal printing temperature of $203{ }^{\circ} \mathrm{C}$.
\end{abstract}

Index Terms - 3D printing, 4D printing, Shape Memory Polymer, Smart materials.

\section{INTRODUCTION}

\section{A. Overview}

Recent advances in additive manufacturing (3D printing) allows researchers to create complex shapes that were previously impossible with the traditional methods. 3D printing is an additive manufacturing process that allows the creation of complex geometries. The manufactured object is produced layer by layer using information from a computer aided design (CAD) file. Recent developments in technology have enabled printing with two different materials to create composites having a variety of scientific and technological applications. A new aspect in additive manufacturing that is coming up is 4D printing [7].

$4 \mathrm{D}$ printing is $3 \mathrm{D}$ printing but with an added dimensional transformation over time using smart materials. The smart material used can be transformed or programmed from one shape to another in response to external stimuli such as heat, light, current or magnetism [1]. 4D is an advancement of 3D where 3D shapes are pushed into development of equipment, deformation of stimuli and conformity to

Published on October 9, 2020.

Francis Irungu Maina, Kenya.

(e-mail: mainaf.maina@gmail.com)

Nahashon Osinde, Kenya.

Japheth Ka'pesha Odira, Kenya.

Patrick Kariuki Wanjiru, Kenya

Margaret Wanjiku Mwangi, Kenya. mathematically modelled variations [6]. The research direction in 4D printing can be summarized in Fig. 1.

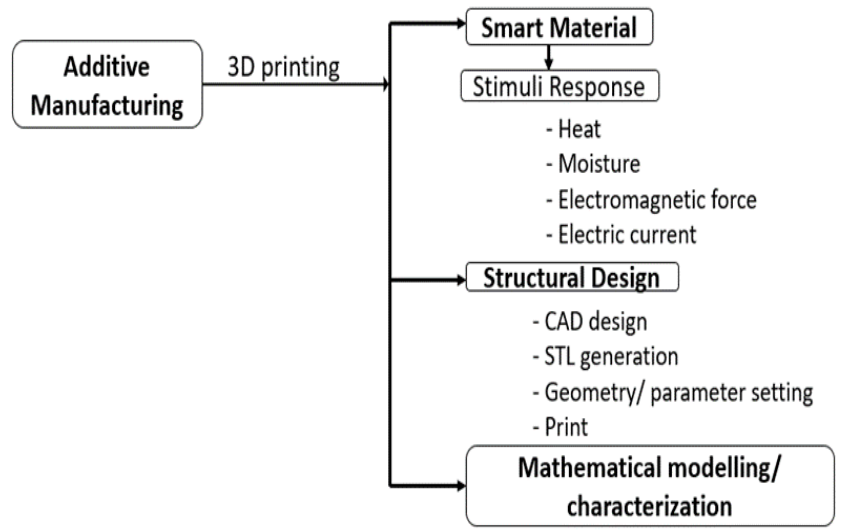

Fig. 1. Research direction in 4D printing.

Materials to be used in 4D printing are not as diversified and mainly the material selection depends on the printer available. 4D printers form a specialized subset of 3D printers including inkjet cure [2], [3], Fused deposition Modelling (FDM) [4,5], stereolithography [9], laser printing [10] and selective laser melting (SLM) [11], [23]. When dealing with $4 \mathrm{D}$ printing, the focus is mainly on deformation mechanism, geometry [12], stress distribution, pattern design [13] and recovery sequence [14]. One major classification of smart materials used in 4D printing is according to thermal responsiveness: either shape memory effect (SME) [15] or the shape change effect (SMC) [16]. SME materials are further divided into shape memory alloys (SMA), shape memory polymers (SMPs) and shape memory hybrids (SMH), shape memory gel (SMG) and shape memory ceramics (SMC). SMPs are made up of a monomer and a crosslinker. The monomer is the soft component (switching segment). It is made up of shorter chains to enable large plastic deformation into a temporary shape at temperatures above the glass transition temperature. The crosslinker serves as the hard component (net-points) [17]. SMPs are favorable due to their glass transition temperature, $\left(T_{g}\right)$, usually higher than the operating temperatures. They have capacity to be programmed under certain mechanical treatment and specific heat above $T_{g}$ and then recover the original shape when cooled at no loading [15], [22]. 4D printing (through selective design) helps optimize the shape recovery (at lower temperatures and higher speed) while focusing on achieving a specific task like gripping.

\section{B. Application}

4D printing has numerous applications. One use is targeted drug delivery to specific locations in the human 
body. The shapes can be stimulated by the body temperature to soften or harden selectively in order to enhance the delivery. Some of these designs include a 4D printed scent [18], 4D nerve guidance conduit [19], and 4D printed scaffold [20]. The smart designs can also be used for soft actuation. Daerden [21] describes a MCkibben type porous silicon elastomer matrix actuated by heat through resistive heating and used for lifting (up to 1700 times its weight).

The use of SMP on artificial muscle to keep the rigid shape even without continuous air supply was investigated by Choong [24]. It was reported that when heated above glass transition temperature ( $\mathrm{Tg})$, SMP entered a soft state and the muscle could then be used as conventional artificial muscles. After the actuator reached its desired length and heat was released, the SMP became rigid and the artificial muscle would be fixed in a stiff and actuated state. This shows an existing trade-off between the printing speed and the surface roughness and relative error as in the Table 1.

TABLE I: PRINTING TRIALS AT DIFFERENT TRANSITION TEMPERATURE

\begin{tabular}{cccccc}
\multicolumn{6}{c}{ AND RETRACT SPEED [24] } \\
\hline \multirow{2}{*}{ Trial } & $T_{g}$ & $v_{e}$ & $\begin{array}{c}\text { Density } \\
\left(\mathrm{g} / \mathrm{cm}^{3}\right)\end{array}$ & $\begin{array}{c}\text { Relative } \\
\text { Error }\end{array}$ & $\begin{array}{c}\text { Surface } \\
\text { Roughness }(\mu \mathrm{m})\end{array}$ \\
\hline 1 & $220^{\circ} \mathrm{C}$ & $90 \mathrm{~mm} / \mathrm{s}$ & 0.839 & 0.0113 & 3.073 \\
2 & $220^{\circ} \mathrm{C}$ & $150 \mathrm{~mm} / \mathrm{s}$ & 0.788 & 0.0204 & 3.575 \\
3 & $250^{\circ} \mathrm{C}$ & $90 \mathrm{~mm} / \mathrm{s}$ & 0.893 & 0.0225 & 6.191 \\
4 & $250^{\circ} \mathrm{C}$ & $150 \mathrm{~mm} / \mathrm{s}$ & 0.887 & 0.0258 & 5.101 \\
\hline
\end{tabular}

An increase in speed and printing temperature resulted in a subsequent increase in density, relative error and surface roughness. Also, heat transfer from the printed SMP to the ambient air is insufficient to cool it down to below glass transition temperature $\left(T_{g}=45{ }^{\circ} \mathrm{C}\right.$ in this case $)$ and contact between extruder and deposited SMP lead to heat transfer and easily heat the already printed SMP to a soft state which largely affect printing quality [24].

Further analysis on the nonlinearity of the model was made by [25]. The model put into consideration the internal friction by considering the fact that the creep recovery where there is no load following loading with the strain (creep), $\varepsilon_{e}$, has some residual irrecoverable strain $\varepsilon_{s}$. The thermal expansion in this case is considered to be independent of the mechanical behaviour. This relationship is modelled as

$$
\dot{\varepsilon}=\dot{\sigma}_{E}+\sigma_{\mu}-\varepsilon-\varepsilon_{s} \lambda+\alpha T
$$

where $\sigma, \varepsilon$ and $T$ represents the stress, strain and temperature respectively.

In the most recent $4 \mathrm{D}$ printing and modelling technologies, the 3D structures are actively transformed in response to some external stimuli over time. They have been reported to recover strain of over $400 \%$ where the recovery is expected to transpire in a nonlinear manner and with the majority of the recovery occurring within minutes of reaching the glass transition temperature [26], [32]. In the Multi-material additive manufacturing systems, the $3 \mathrm{D}$ shapes memory structures are printed through the additive process. A computer aided design (CAD) is first sliced through the CURA software into a series of closely spaced yet horizontal 2D digital images [30]. These are then transmitted to a code (G-code) readable by the printer. In most cases, the printer uses artificial intelligence to compute the simplest route for printing and does so layer by layer in what is referred to as the bottom-up method.

\section{Design Considerations}

The static behaviour and study of important design considerations including local deformation, shape fixity and free recovery rate is expected to follow the phase transition as represented in Fig. 2.

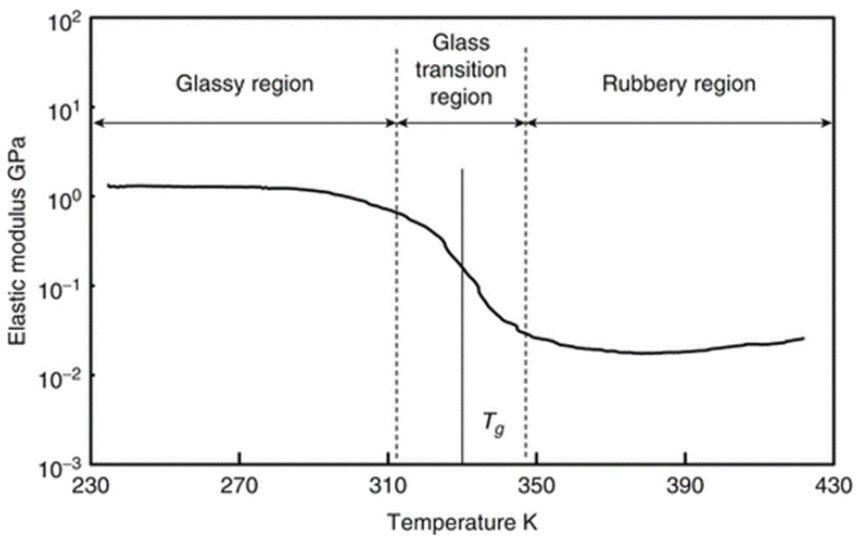

Fig. 2. Graph of Elastic Modulus against Temperature (Phases of SMP material) [31]

Before mechanical modelling and testing, some fundamental properties of the SMP ought to be assessed. The filament used in this project was the polyurethane of diameter $1.75 \mathrm{~mm}$, a glass temperature of 55 degrees Celsius and a roll $300 \mathrm{~g} / \mathrm{roll}$. Referring to the test conducted by [27], [28], as the material heats up and changes phase, the movement of the probe tracks the deflection of the material with respect to temperature. The points at which softening occurs and the subsequent thermal expansion can be tracked from the resultant graph, Fig. 3. The conducted experiment resulted in the $T_{g}$ of $50-58{ }^{\circ} \mathrm{C}$ with the ultimate being 58 degrees.

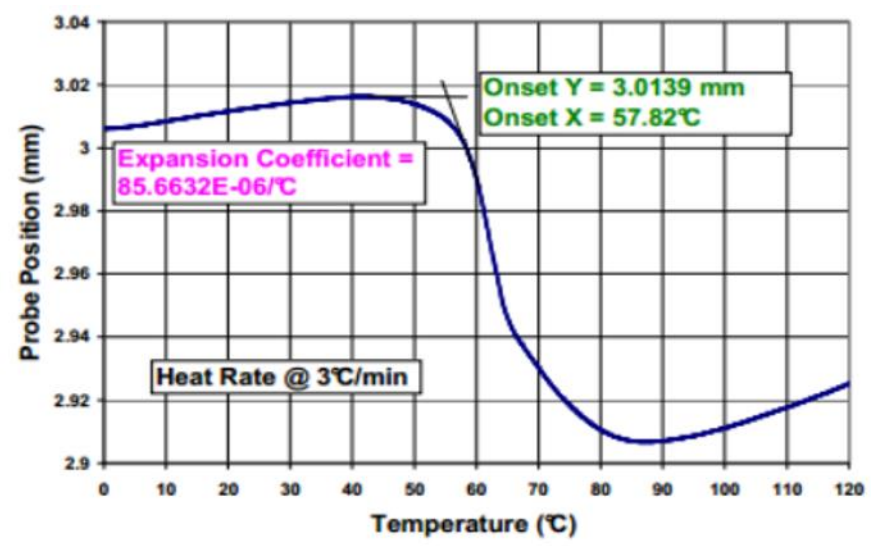

Fig. 3. Tg Measurement Using TMA [28].

\section{CAD MODELLING}

Different considerations during the design were made including the need for gripping upon displacement of gripper arms. From the preliminary test conducted in Ultimaker 3D Printer, there was no support material for SMP filament. This meant that the gripper could only be made as a two tong hence limiting the capacity to exercise with complex smart shapes. Also, there was the limitation of 
space forcing the gripper to be relatively small. A trade-off had to be made between the gripper thickness and grip strength while optimizing the speed of gripping.

\section{A. Design Flow}

The following is the flow diagram shows the process of 4D printing

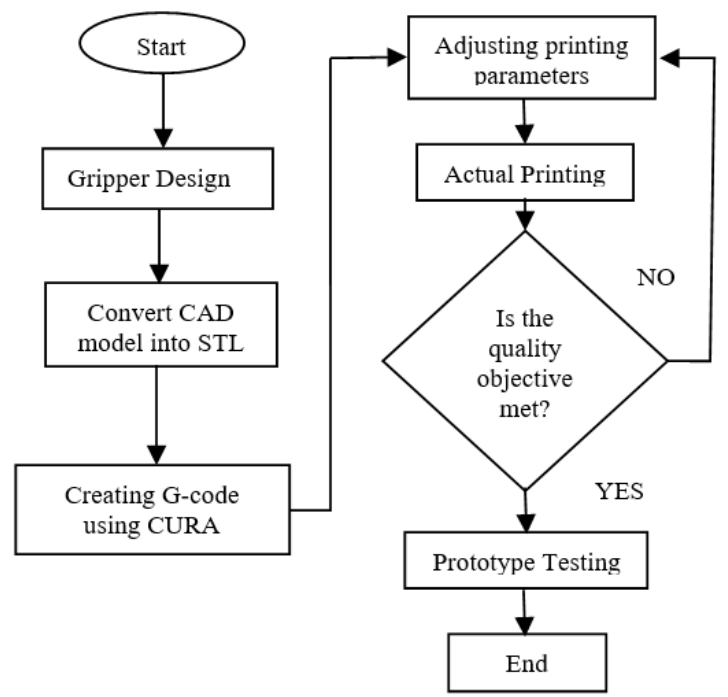

Fig. 4. Design Flow chart.

\section{B. Parameter Setting for Printing}

An SMP filament $(1.78 \mathrm{~mm}$ diameter) and Ultimaker $23 \mathrm{D}$ printer (one nozzle) were used in this research [24]. Before printing, parameters were set in the CURA software including the infill, the support selection, layer pattern, printing speed and rack structure [29]. Owing to the lack of dissolvable (easily removable) support material for printing shapes with structures requiring support, a simple structure was designed initially. The orientation of the 3D shape was done in the Inventor software by selecting the Ultimaker 2 as the designated printer and the structure orientation selected as lying flat on the bed as in Fig. 5.

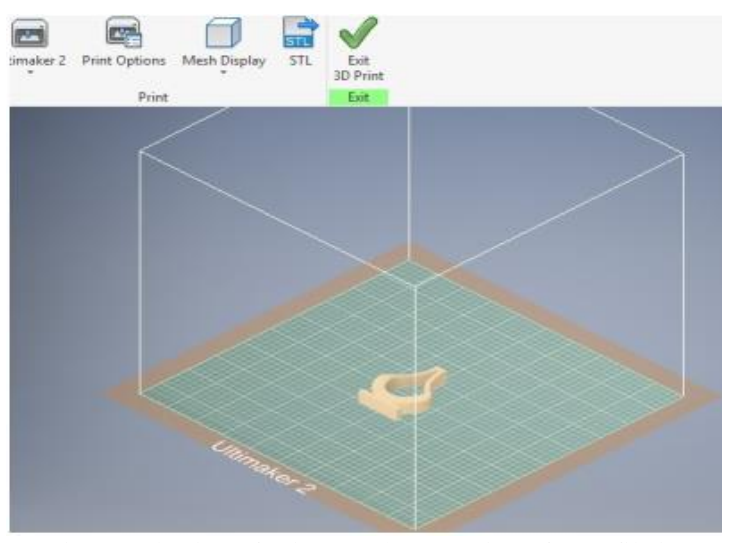

Fig. 5. Selection of Printer and Generation of STL file in Inventor.

The manufacturer of the SMP filament material recommended a nozzle temperature of $195-210{ }^{\circ} \mathrm{C}$ and no build plate temperature. An experiment to determine the optimal printing speed and printing temperature was conducted. Taguchi's approach [27] was adopted to reduce the number of experiments based on well-defined guidelines instead of doing a full factorial design of experiments. An orthogonal array stipulated the way of conducting the minimal number of experiments which could give full information of all the factors that affect the performance parameter. Using Taguchi, the number of experiments were reduced to a practical level by selecting a small set of possibilities. This is useful when all the possible combinations of inputs result in a huge impractical number of experiments. The Taguchi method was run on the Minitab software.

\section{DESIGN OF EXPERIMENT}

Different parameters were assessed in this case given the fact that the best printing properties were already not known. The first step involved printing a stencil (vertical bar) where, after every few millimeters of printing, the parameters for the infill, sweep, retract length and nozzle temperature were changed. The resulting $3 \mathrm{D}$ printout was as in the Fig. 6.

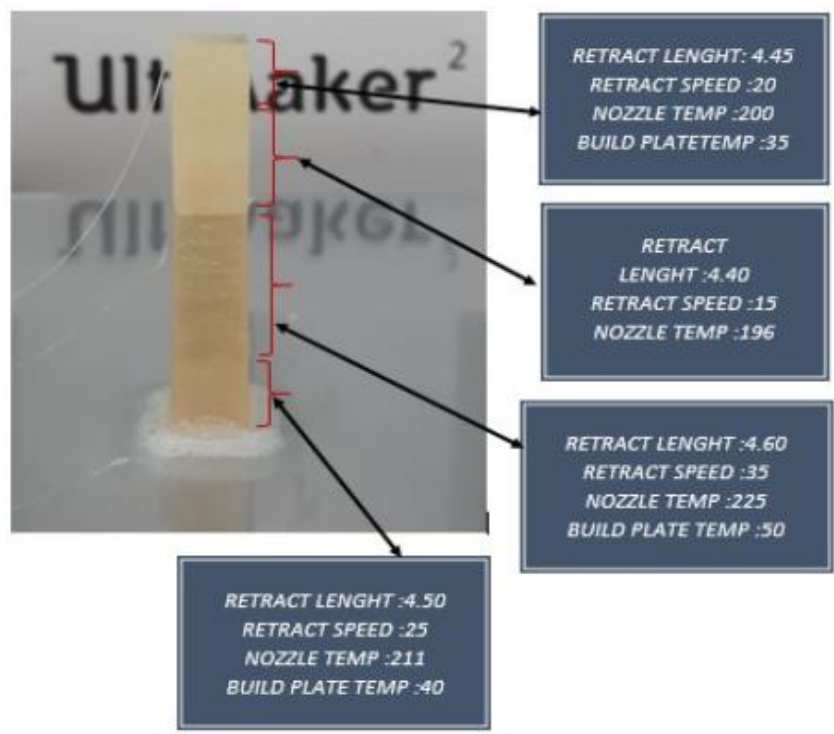

Fig. 6. Parameter Testing on Ultimaker 2 Printer Prior to Actual Printing.

In this method, the top layer of the vertical stencil with the parameters of 4.45 retract length, 20-30 retract speed, 195-210 ${ }^{\circ} \mathrm{C}$ nozzle temperature and a build temperature of $\leq 35^{\circ} \mathrm{C}$ resulted in the best print quality. A higher build plate temperature caused the printed material to stick to the plate hence causing breakages during retrieval. The retract speed, if increased, caused uncured layers within the structure making the slices non uniform. This also occurred when the retract speed was high and residue fibres occurred all over the infill pattern pores. A more scientific way of defining the optimal parameters had to be used to lower the parameter range.

\section{A. First Design of Experiment (DOE)}

In the first design of experiment (DOE) the printing speed was varied from $30 \mathrm{~mm} / \mathrm{s}$ to $50 \mathrm{~mm} / \mathrm{s}$ and the temperature from $195-210{ }^{\circ} \mathrm{C}$ according to Table II and a rectangular stencil was printed in each case. 
TABLE II: FIRST DESIGN OF EXPERIMENT

\begin{tabular}{ccc}
\hline Experiment Number & Speed $(\mathrm{mm} / \mathrm{s})$ & Temperature $\left({ }^{\circ} \mathrm{C}\right)$ \\
\hline 1 & 40 & 195 \\
2 & 40 & 203 \\
3 & 40 & 210 \\
4 & 30 & 195 \\
5 & 30 & 203 \\
6 & 30 & 210 \\
7 & 50 & 210 \\
8 & 50 & 203 \\
9 & 50 & 195 \\
\hline
\end{tabular}

It was found out that a temperature of $195{ }^{\circ} \mathrm{C}$ was not ideal for the gripper in terms of its dynamic characteristics. By doing this type of DOE, the effect of infill density on the dynamic properties of the gripper was not considered.

\section{B. Second Design of Experiment (DOE)}

Similar to the first DOE, Taguchi's L9 orthogonal array was used. Out of a possible 27 experiments, only 9 were performed. The temperature was varied from $195-210{ }^{\circ} \mathrm{C}$, the infill density from $15 \%$ to $60 \%$ and the speed from 20 $\mathrm{mm} / \mathrm{s}$ to $60 \mathrm{~mm} / \mathrm{s}$ as shown in Table III. The base speed was set at $40 \mathrm{~mm} / \mathrm{s}$, where a percentage of 50 would imply a speed of $20 \mathrm{~mm} / \mathrm{s}$.

TABLE III: SECOND DESIGN OF EXPERIMENT

\begin{tabular}{cccc}
\hline Experiment Number & Temperature $\left({ }^{0} \mathrm{C}\right)$ & Infill $(\%)$ & Speed (\%) \\
\hline 1 & 195 & 15 & 50 \\
2 & 195 & 38 & 100 \\
3 & 195 & 60 & 150 \\
4 & 203 & 38 & 50 \\
5 & 203 & 60 & 100 \\
6 & 203 & 15 & 150 \\
7 & 210 & 60 & 50 \\
8 & 210 & 15 & 100 \\
9 & 210 & 38 & 150 \\
\hline
\end{tabular}

Using these parameters, small beads were printed as shown in the Fig. 7. These were used to visually assess the print quality.

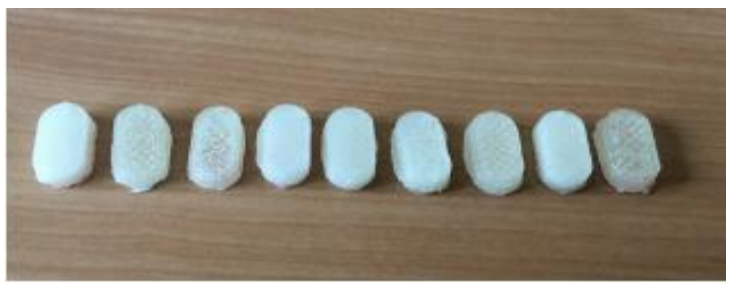

Fig. 7. Beads printed in the second design of experiment.

From this DOE a printing speed of $40 \mathrm{~mm} / \mathrm{s}$, an infill of $38 \%$ and a printing temperature of $203{ }^{\circ} \mathrm{C}$ was selected as the one producing the best print quality through visual inspection. This prompted the printing of the first gripper prototype.

\section{Gripper Printing Prototype}

A gripper model was designed in Autodesk 360. It had two fingers with grooves to enable better gripping of an object as shown in Fig. 8.

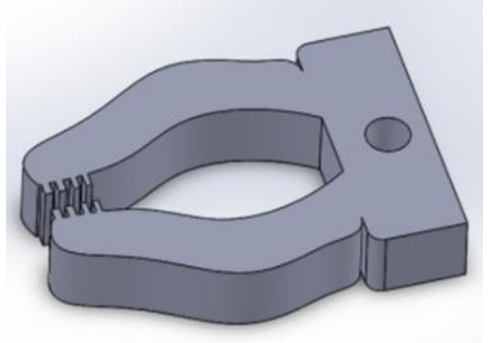

Fig. 8. Last Gripper Model.

A scaled down model prototype of the gripper was printed to assess the print quality and functionality, but a stringing effect was encountered. As observed in Fig. 9, string-like structures formed when the printer's nozzle travelled from one finger to the next. These lowered the print quality and increased the post processing time of the print work.

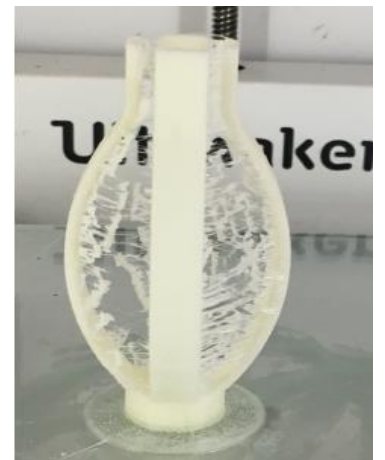

Fig. 9. First prototype of gripper having string like structures.

Melted filament still oozed out when the nozzle made a travel movement between the fingers, as in Fig. 10 (a), which was the cause of the stringing effect. The retraction settings had to be tweaked so that when the nozzle made a travel movement between two fingers the filament was pulled back slightly. The parameters to be optimized were the retraction speed and length. The retraction parameters were tweaked by varying the retraction speed from 20 to 45 $\mathrm{mm} / \mathrm{s}$ and the retraction length from $4.5 \mathrm{~mm}$ to $6.5 \mathrm{~mm}$ in $0.5 \mathrm{~mm}$ increments. Retraction speed of $30 \mathrm{~mm} / \mathrm{s}$ and retraction length of $4.5 \mathrm{~mm}$ produced less pronounced string like structures but did not eliminate them completely. At a higher temperature, $210{ }^{\circ} \mathrm{C}$ there was less stringing but at a cost of quality as shown in Fig. 10(b).
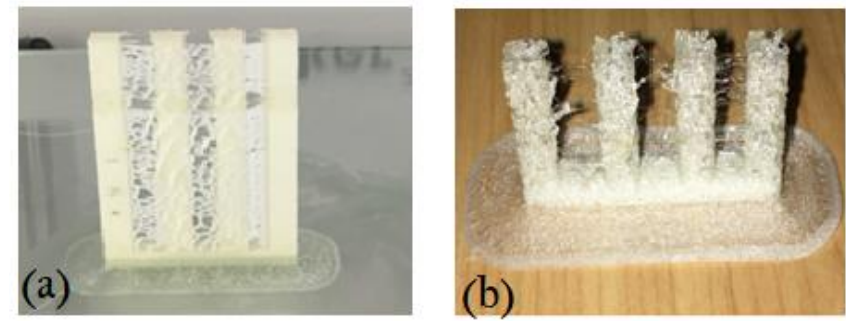

Fig. 10. (a) Stringing effect during tweaking of parameters (b) Less stringing effect but poor quality.

The retraction parameters mentioned were used and followed by a post-processing with a sandpaper to obtain a smooth surface finish in the subsequent printing. 


\section{Actual Gripper Printing}

The design in Fig. 13 was settled upon as the best quality after multiple tests from different designs. More analysis was conducted by printing grippers at different temperature and infill parameters. The resultant designs were as presented in the Fig. 11, 12 and 13. The first printed prototype of the gripper (Fig. 11), even though satisfactorily met the gripping function, consumed more printing material and thus was not economical. The second gripper (Fig. 12) also performed the gripping function but in its fully stretched limit the recovering forces were weak. The third design (Fig. 13) was selected as the final design. It performed the gripping function better than the earlier designed prototypes. The simplicity of the model consumed less printing material and robustness in terms of shape recovery and fixity was guaranteed.

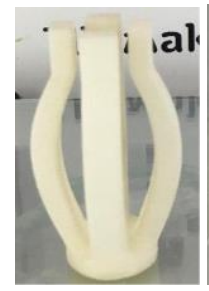

Fig. 11. First gripper

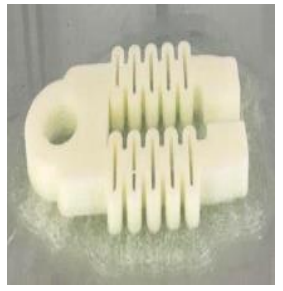

Fig. 12. Second gripper

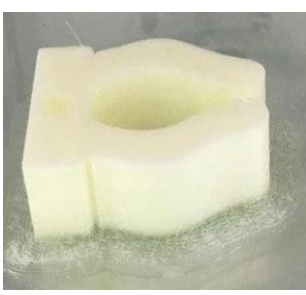

Fig. 13. Third gripper

\section{E. Shape Recovery}

The shape recovery experiments were carried out in a water-bath as shown in Fig. 14. The water was heated to a temperature $55^{\circ} \mathrm{C}$ which was slightly higher than the glass transition temperature of SMP material. The SMP material softened after being heated in the water-bath for some time and the gripper was manually deformed to an open position. It was left to cool at room temperature and became rigid. The gripper was then put inside the water-bath at $45{ }^{\circ} \mathrm{C}$ which is the glass transition temperature. The gripper recovered its initial shape. Fig. 15 ((a)-(d)) shows the shape transition steps of the gripper.

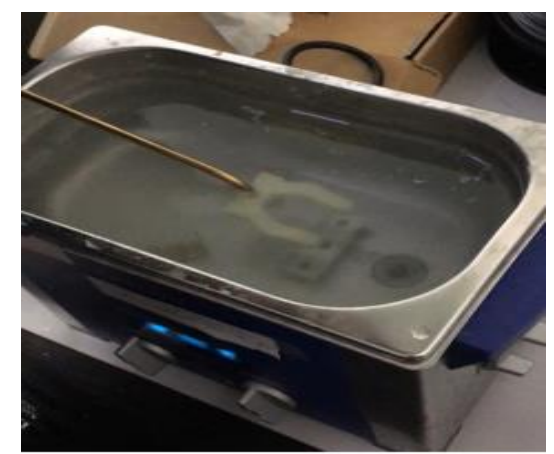

Fig. 14. Gripper heat actuation through hot water.

The following final printed models were used in the characterization.

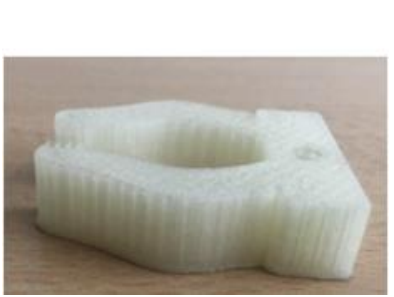

(a) 3D image

of the gripper

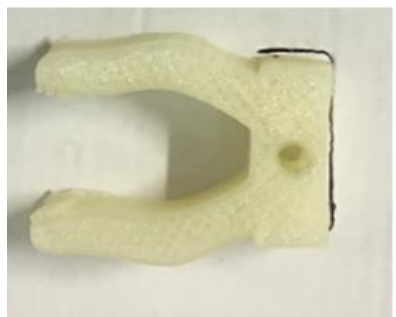

(c) Open gripper after deformation upon heating beyond $T_{g}$

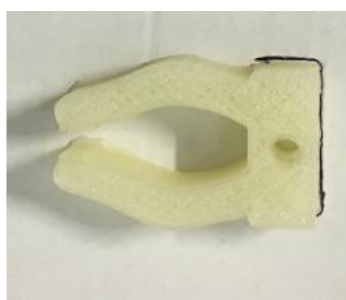

(b) Initial gripper shape before manual deformation

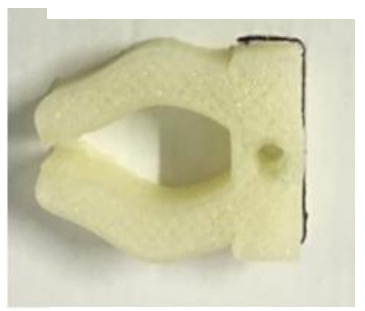

(d) Gripper recovered shape upon reheating to $T_{g}$
Fig. 15.

The object gripping property was assessed as the gripper was reheated to $T_{g}$ in Fig. 15 (d). Fig. 16 shows the gripper grasping an object.

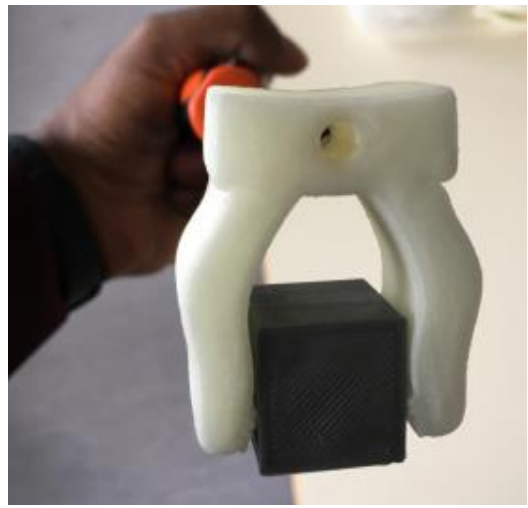

Fig. 16. Gripper grasping an object

This proved that the SMP can easily be deformed without breaking when heated beyond the glass transition temperature and has the capacity to recover its initial shape when reheated to the glass transition temperature.

\section{CHARACTERIZATION}

\section{A. Shape Recovery Characterization}

Further experiments were carried out on 4 grippers based on the third prototype design (Fig. 13). Each gripper was printed using different parameters and analysis performed to test whether the grippers recover their original shape as expected. To investigate whether the gripper recovered its initial shape, the initial photo of the gripper was overlaid onto the final shape after recovery and a comparison rate of change characterized. The 4 grippers were printed using the following parameters:

- Printing temperature; $203{ }^{\circ} \mathrm{C}$, infill of $38 \%$, printing speed of $40 \mathrm{~mm} / \mathrm{s}$ 
- Printing temperature; $210{ }^{\circ} \mathrm{C}$, infill of $60 \%$, printing speed of $40 \mathrm{~mm} / \mathrm{s}$

- Printing temperature; $195{ }^{\circ} \mathrm{C}$, infill of $20 \%$, printing speed of $40 \mathrm{~mm} / \mathrm{s}$

- Printing temperature; $203{ }^{\circ} \mathrm{C}$, infill of $80 \%$, printing speed of $40 \mathrm{~mm} / \mathrm{s}$

\section{B. Analysis of the Results}

In this section, analysis in terms of shape recovery was done for the four grippers.

\section{Gripper Design 1 (Printing Temperature - $203{ }^{\circ} \mathrm{C}$,} infill - 38\%)

The gripper recovery extent was analyzed by overlying the final image onto the initial image using MATLAB as shown in the Fig 17. This first gripper was printed at a temperature of $203{ }^{\circ} \mathrm{C}$, infill of $38 \%$ and printing speed of $40 \mathrm{~mm} / \mathrm{s}$ and the Fig. 17 (a) represents the image of the printout used for analysis.
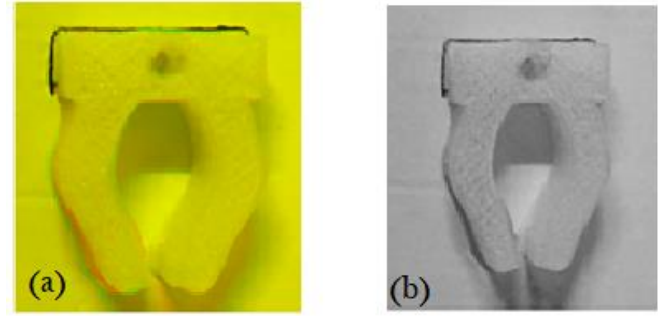

Fig. 17. First gripper with initial and final shape overlaid on top of each other (a) Colored image after analysis and (b) Grey-scale image after analysis.
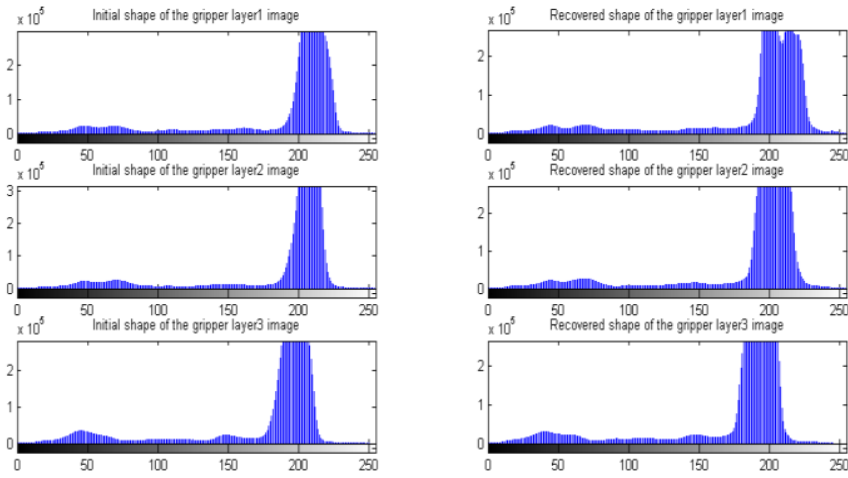

Fig. 18. Histogram showing distribution of RGB values

Fig. 17a and $17 \mathrm{~b}$ show cascaded images of the initial shape of the gripper before gripping and the final shape of the gripper after gripping. They were overlaid on top of each other with the datum base of reference being the underlined mark in black. The scope here was to compare whether the gripper recovered its initial shape after performing the gripping action fully. From Fig. 17, gripper 1 nearly recovered all the initial shape with the exception of a slight variation which was witnessed from the histogram plot Fig. 18. The histogram plots on the left show the image distribution of the initial shape of the gripper based on the "rgb" layer distribution in image processing of MATLAB of which $r$ is red, $g$ is green and $b$ is the blue layer respectively. The plot on the right show the image distribution of the final shape of the gripper. This distribution shows that the recovered shape of the gripper on the right perfectly tracks that of the initial shape only with a slight variation at the peak of the right histogram. From the IMGonline.com using the link https://www.imgonline.com.ua/eng/similaritypercent result.php. The percentage similarity index was $96.93 \%$ for the printing parameters of temperature $203{ }^{\circ} \mathrm{C}$, infill of $38 \%$, printing speed of $40 \mathrm{~mm} / \mathrm{s}$.

\section{Gripper Design 2 (Printing Temperature $-210{ }^{\circ} \mathrm{C}$, infill-60\%)}

The analysis was conducted as described in the first gripper. This second gripper was printed at a temperature of $210{ }^{\circ} \mathrm{C}$, infill of $60 \%$ and printing speed of $40 \mathrm{~mm} / \mathrm{s}$.
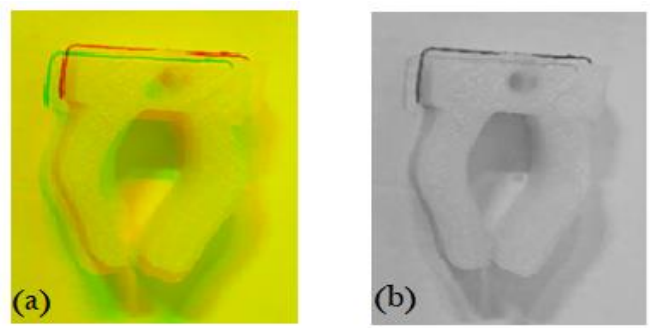

Fig. 19. Second gripper with initial and final shape overlaid on top of each other (a) Coloured image after analysis and (b) Grey-scale image after analysis.
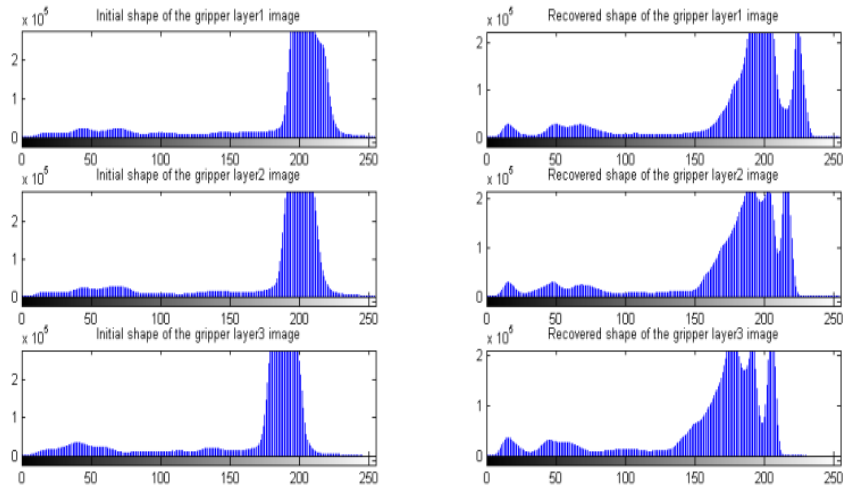

Fig. 20. Histogram showing distribution of RGB values

From the image comparison of Fig. 19a and 19b, the gripper tried to recover its initial shape but did not recover completely. The disparity was observed clearly from the histogram distribution Fig. 20. From the IMGonline.com, the percentage similarity of the picture was $89.23 \%$. This low recovery rate was attributed to the high infill factor and temperature used in printing which made the gripper design more rigid.

\section{Gripper Design 2 (Printing Temperature $-195^{\circ} \mathrm{C}$, infill $-20 \%$ )}

This third gripper was printed at a temperature of $195{ }^{\circ} \mathrm{C}$, infill of $20 \%$ and printing speed of $40 \mathrm{~mm} / \mathrm{s}$.
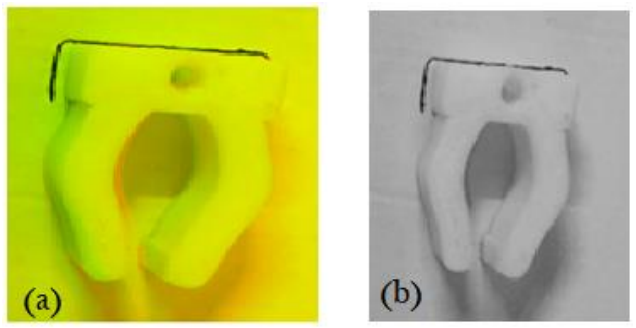

Fig. 21. Third gripper with initial and final shape overlaid on top of each other (a) Coloured image after analysis and (b) Grey-scale image after analysis 

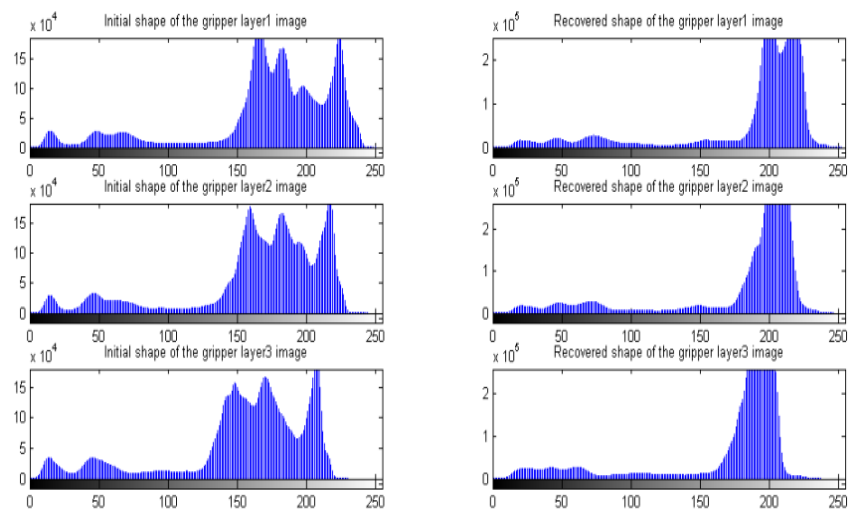

Fig. 22. Histogram showing distribution of RGB values.

From the Fig. 21a and 21b, the gripper assumes its original shape but with only tiny variations as seen in the slight red colour highlight. The histogram data, Fig. 22, showed uniform distribution of the data across all the layers and the recovered shapes slightly varied from the initial shape. From the IMGonline.com the percentage similarity of the picture was $92.67 \%$ The increase in the shape recovery index was attributed to the lower infill rate and printing temperature which made the design more porous.

\section{Gripper Design 2 (Printing Temperature $-203^{\circ} \mathrm{C}$, infill $-80 \%$ )}

The analysis is as described earlier for the first gripper. This fourth gripper was printed at a temperature of $203{ }^{\circ} \mathrm{C}$, infill of $80 \%$ and printing speed of $40 \mathrm{~mm} / \mathrm{s}$.
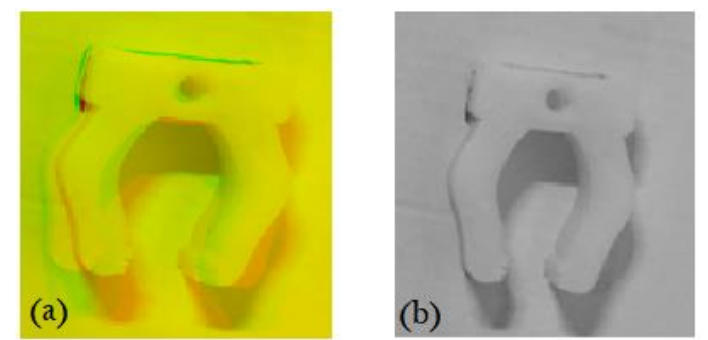

Fig. 23. Fourth gripper with initial and final shape overlaid on top of each other (a) Colored image after analysis and (b) Grey-scale image after analysis.

The fourth gripper, Fig. 23 could not recover completely to its initial shape. The histogram data, Fig. 24, show uniform distribution of the data across all the layers and the recovered shapes variation from the initial shape. From the IMGonline.com the percentage similarity of the print was $86.6 \%$. This effect was majorly attributed to the thickness of the gripper, and the printing infill density.
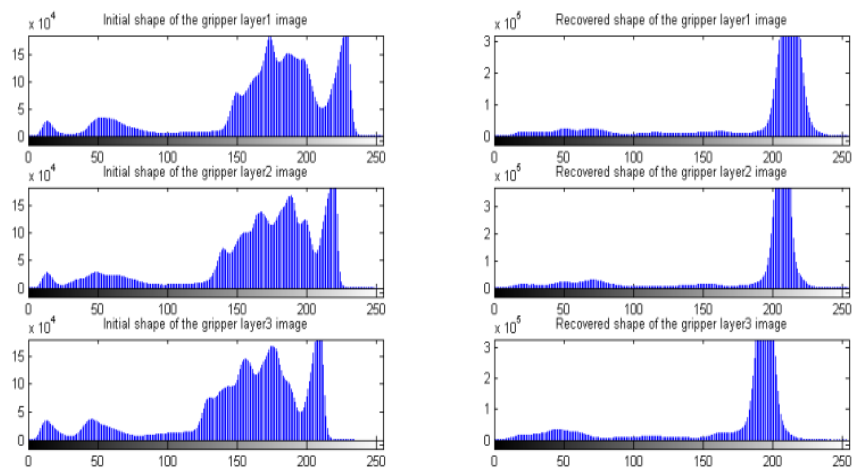

Fig. 24. Histogram showing distribution of RGB values.

\section{DISCUSSION}

At a temperature above the glass transition temperature, $T_{g}$, the Shape Memory Polymer (SMP) easily changed the form when low stress was applied. The material remained rigid at temperatures below the glass transition and application of force made it break. In this case the printed shape was first heated at the Glass transition temperature, deformed, and allowed to cool to retain the deformed shape. Upon the reheating at the glass transition temperature the original shape was recovered. This means that the shape recovered naturally without the need for an external force and hence proofing the shape recovery ration of about one.

As a consequence, relative error of printed SMP part was observed to be large in low speed $v_{e}$. If $v_{e}$ was too fast, extrusion of SMP was not sufficient. High extrusion speed led to vibration of the $3 \mathrm{D}$ printing machine. These two reasons resulted in increased relative error at increasing speed $v_{e}$ when the nozzle scanning speed was over $40 \mathrm{~mm} / \mathrm{s}$. There was a trade-off between retract length and nozzle speed. An increase in the retract speed resulted in poor infill of the material and hence a weaker structure. Equally, the build plate temperature was set below $35^{\circ} \mathrm{C}$ to help a better printout removal. An increase in the build plate temperature resulted in the material sticking and removal meant that some part was to be broken. It can be concluded that the slower the scanning speed, the denser the part will be within the working temperature range (196-210 $\left.{ }^{\circ} \mathrm{C}\right)$. Upon successful printing the shape fixity ratio and shape recovery ratio were assessed.

The varying performances of the four grippers can be attributed to the different infill percentages. The lower the infill, the higher the recovery rate due to the low stiffness of the gripper. The gripper with higher infill had less recovery due to the high stiffness. However, the recovery below the optimal infill rate and temperature are low which was attributed to too much flexibility making the shape not strong enough to fully recover the original shape. From the results, gripper 1 and 3 had better recovery rate, $96.96 \%$ and $92.67 \%$ respectively. Gripper 1 had the best recovery rate since it was printed at a temperature of $203^{\circ} \mathrm{C}$ which was optimal.

\section{CONCLUSION}

A 4D smart gripper model based on SMP was designed, printed and actuated by heat as the stimuli. The 3D printer set up and optimization of printing parameters to give the best print quality was studied. An optimal temperature of $203{ }^{\circ} \mathrm{C}$, infill density of $38 \%$ and printing speed of $40 \mathrm{~mm} / \mathrm{s}$ gave the best print quality of the gripper. Characterization of the shape recovery of the grippers was conducted and the results analyzed. The gripper printed at the optimal parameters gave the best recovery of $96.93 \%$. This study demonstrated the shape recovery of SMP smart material 4D printout and opens the possibility of utilizing design and the optimal printing parameters to make usable structures. An interesting topic that should be further emphasized upon is topology optimization since it finds crucial application in the field of design. 


\section{REFERENCES}

[1] Wagermaier, W., Kratz, K., Heuchel, M., \& Lendlein, A. (2009). Characterization methods for shape-memory polymers. In Shapememory polymers (pp. 97-145). Springer, Berlin, Heidelberg.

[2] Ge, Q., Qi, H. J., \& Dunn, M. L. (2013). Active materials by fourdimension printing. Applied Physics Letters, 103(13), 131901.

[3] Liu, Y., Shaw, B., Dickey, M. D., \& Genzer, J. (2017). Sequential self-folding of polymer sheets. Science Advances, 3(3), e1602417.

[4] Liu, Y., Zhang, W., Zhang, F., Lan, X., Leng, J., Liu, S., \& Chou, T. W. (2018). Shape memory behavior and recovery force of 4D printed laminated Miura-origami structures subjected to compressive loading. Composites Part B: Engineering, 153, 233-242.

[5] Zhang, Q., Yan, D., Zhang, K., \& Hu, G. (2015). Pattern transformation of heat-shrinkable polymer by three-dimensional (3D) printing technique. Scientific reports, 5, 8936

[6] Tibbits, S., McKnelly, C., Olguin, C., Dikovsky, D., \& Hirsch, S (2014). 4D Printing and universal transformation.

[7] Pei, E., \& Loh, G. H. (2018). Technological considerations for 4D printing: an overview. Progress in Additive Manufacturing, 3(1-2), 95-107.

[8] Dadbakhsh, S., Speirs, M., Kruth, J. P., Schrooten, J., Luyten, J., \& Van Humbeeck, J. (2014). Effect of SLM parameters on transformation temperatures of shape memory nickel titanium parts. Advanced Engineering Materials, 16(9), 1140-1146.

[9] Hager, M. D., Bode, S., Weber, C., \& Schubert, U. S. (2015). Shape memory polymers: Past, present and future developments. Progress in Polymer Science, 49, 3-33.

[10] Voit, W., Ware, T., Dasari, R. R., Smith, P., Danz, L., Simon, D., \& Gall, K. (2010). High-strain shape-memory polymers. Advanced functional materials, 20(1), 162-171.

[11] Treloar, L. R. G. (1975). The physics of rubber elasticity. Oxford University Press, USA

[12] Wu, W., \& You, Z. (2011). A solution for folding rigid tall shopping bags. Proceedings of the Royal Society A: Mathematical, Physical and Engineering Sciences, 467(2133), 2561-2574.

[13] Safranski, D. L., \& Gall, K. (2008). Effect of chemical structure and crosslinking density on the thermo-mechanical properties and toughness of (meth) acrylate shape memory polymer networks. Polymer, 49(20), 4446-4455.

[14] Choi, J. W., Kim, H. C., \& Wicker, R. (2011). Multi-materia stereolithography. Journal of Materials Processing Technology, 211(3), 318-328.

[15] Anand, L., Ames, N. M., Srivastava, V., \& Chester, S. A. (2009). A thermo-mechanically coupled theory for large deformations of amorphous polymers. Part I: Formulation. International Journal of Plasticity, 25(8), 1474-1494.

[16] Ames, N. M., Srivastava, V., Chester, S. A., \& Anand, L. (2009). A thermo-mechanically coupled theory for large deformations of amorphous polymers. Part II: Applications. International Journal of Plasticity, 25(8), 1495-1539.

[17] Hetnarski, R. B. (Ed.). (2014). Encyclopedia of thermal stresses. Springer Netherlands.

[18] Malachowski, K., Breger, J., Kwag, H. R., Wang, M. O., Fisher, J. P., Selaru, F. M., \& Gracias, D. H. (2014). Stimuli-responsive theragrippers for chemomechanical controlled release. Angewandte Chemie International Edition, 53(31), 8045-8049.

[19] Miao, S., Cui, H., Nowicki, M., Xia, L., Zhou, X., Lee, S. J., ... \& Zhang, L. G. (2018). Stereolithographic 4D bioprinting of multiresponsive architectures for neural engineering. Advanced biosystems, 2(9), 1800101.

[20] Shian, S., Bertoldi, K., \& Clarke, D. R. (2015). Dielectric elastomer based "grippers" for soft robotics. Advanced Materials, 27(43), 68146819.

[21] Daerden, F., \& Lefeber, D. (2002). Pneumatic artificial muscles: actuators for robotics and automation. European journal of mechanical and environmental engineering, 47(1), 11-21.

[22] Yang, Y., Chen, Y., Wei, Y., \& Li, Y. (2016). 3D printing of shape memory polymer for functional part fabrication. The International Journal of Advanced Manufacturing Technology, 84(9-12), 2079. 2095.

[23] Koch, L., Deiwick, A., \& Chichkov, B. (2018). Laser-Based Cell Printing, in 3D Printing and Biofabrication.

[24] Choong, Y. Y. C., Maleksaeedi, S., Eng, H., Wei, J., \& Su, P. C. (2017). 4D printing of high performance shape memory polymers using stereolithography. Materials \& Design, 126, 219-225.

[25] Tobushi, H., Okumura, K., Hayashi, S., \& Ito, N. (2001) Thermomechanical constitutive model of shape memory polymer. Mechanics of materials, 33(10), 545-554
[26] Hu, J., Zhu, Y., Huang, H., \& Lu, J. (2012). Recent advances in shape-memory polymers: Structure, mechanism, functionality, modeling and applications. Progress in Polymer Science, 37(12) 1720-1763.

[27] Ge, Q., Sakhaei, A. H., Lee, H., Dunn, C. K., Fang, N. X., \& Dunn, M. L. (2016). Multimaterial 4D printing with tailorable shape memory polymers. Scientific reports, 6, 31110 .

[28] Yang, Y., Chen, Y., Wei, Y., \& Li, Y. (2016). 3D printing of shape memory polymer for functional part fabrication. The International Journal of Advanced Manufacturing Technology, 84(9-12), 20792095.

[29] Tani, J., Takagi, T., \& Qiu, J. (1998). Intelligent material systems: application of functional materials

[30] Ariffin, M. K. A., Sukindar, N. A., Baharudin, B. T. H. T., Jaafar, C. N. A., \& Ismail, M. I. S. (2018, January). Slicer Method Comparison Using Open-source 3D Printer. In IOP Conference Series: Earth and Environmental Science (Vol. 114, p. 012018).

[31] Tobushi, H., Hara, H., Yamada, E., \& Hayashi, S. (1996). Thermomechanical properties in a thin film of shape memory polymer of polyurethane series. Smart Materials and Structures, 5(4), 483.

[32] Couchman, P. R. (1978). Compositional variation of glass-transition temperatures. 2. Application of the thermodynamic theory to compatible polymer blends. Macromolecules, 11(6), 11561161.Development and Research, ed. Chen Dan, pp. 359-366, vol. 26, Singapore, 2011 